\title{
Sudden Frequency Deviations due to Immediate Effect of Geomagnetic Sudden Commencements
}

\author{
J. Hanumath SASTRI* and C.V. SUbrahmANYAM \\ Radio Science Division, National Physical Laboratory, \\ New-Delhi, India
}

(Received June 24, 1974; Revised August 13, 1974)

\begin{abstract}
HF Doppler data over two long low latitude radio paths (ATA-Haringhata and JJY-Haringhata) for the period June, 1966 to December 1972 has been examined for effects of sudden commencements. It is noticed that for the same type of sudden commencement, i.e., $\mathrm{SC}(+)$, four distinct types of ferequency deviations exist, designated as $\operatorname{SCFD}(+), \operatorname{SCFD}(-), \operatorname{SCFD}(+-)$ and SCFD $(-+)$. The occurrence and the nature of the frequency deviation effect due to sudden commencement is independant of the amplitude of the sudden commencement. A positive correlation between the amplitude of the maximum positive frequency deviation and maximum negative frequency deviation is noticed in composite events: SCFD (+-) and SCFD $(-+)$.
\end{abstract}

\section{Introduction}

One of the established experimental techniques for investigating the ionospheric response to transient phenomena like solar flares and geomagnetic sudden commencements is the HF Doppler sounder technique developed by OGAWA (1958), Fenwick and Villard (1960) and Watts and Davies (1960). Davies (1962), Kanellakos and Villard (1962), Chan et al. (1962) and Agy et al. (1965) have reported their results regarding the effect of storm sudden commencements on the ionosphere using the above technique. However, the effect has not been studied in detail over low latitude radio paths but for the recent effort of Huang et al. (1973) who studied the Doppler data of a short low latitude path for effects of sudden commencements. The present communication deals with the results of a similar attempt using HF Doppler data over two relatively long (path length $1000 \mathrm{~km}$ ) low latitude radio paths: ATA (10 $\mathrm{MHz}$ )-Haringhata (path length $1290 \mathrm{~km}$ ) and JJY $(10 \mathrm{MHz})$-Haringhata (path length $5700 \mathrm{~km}$ ) and geomagnetic data from the Hyderabad Geomagnetic Observatory. The frequency of transmission of both the ATA and JJY stations is $10 \mathrm{MHz}$. Geomagnetic storm sudden commencements that occurred between June 1966 to December 1972 have been used for the study of possible ionospheric effects. The Geomagnetic and Geographic co-ordinates of the locations

\footnotetext{
* Present address: Indian Institute of Astrophysics, Kodaikanal, India.
} 
Table 1.

\begin{tabular}{lccccc}
\hline \multirow{2}{*}{ Name of Station } & \multicolumn{2}{c}{ Latitude: } & & \multicolumn{2}{c}{ Longitude: } \\
\cline { 2 - 3 } \cline { 5 - 6 } \cline { 5 - 6 } & Geographic & Geomagnetic & & Geographic & Geomagnetic \\
\hline ATA, Delhi & $28^{\circ} 38^{\prime} \mathrm{N}$ & $19^{\circ} 11^{\prime} \mathrm{N}$ & & $77^{\circ} 13^{\prime} \mathrm{E}$ & $148^{\circ} 55^{\prime} \mathrm{E}$ \\
Haringhata & $2258 \mathrm{~N}$ & $1215 \mathrm{~N}$ & & $8834 \mathrm{E}$ & $15842 \mathrm{E}$ \\
JJY, Tokyo & $3542 \mathrm{~N}$ & $2600 \mathrm{~N}$ & & $13930 \mathrm{E}$ & $20600 \mathrm{E}$ \\
Hyderabad & $1721 \mathrm{~N}$ & $739 \mathrm{~N}$ & & $7828 \mathrm{E}$ & $14857 \mathrm{E}$ \\
\hline
\end{tabular}

of the Haringhata field station, ATA and JJY stations and the Hyderabad Geomagnetic Observatory are given in Table 1.

Between June 1966 to December 1972, 184 storm sudden commencements (SSC) have been observed at the Hyderabad Geomagnetic Observatory. (B.J. SRIvastava, private communication, 1973). Out of these 184 SC's, 24 occurred during the 'off' period of transmissions and power failures at the recording site, while 67 occurred during the maintenance period of the Doppler system. For $33 \mathrm{SC}$ events, poor ionospheric conditions made it difficult to identity the effects. Thus a total of $60 \mathrm{SC}$ events became available to study their effects on frequency deviations over the low latitude radio paths. Distinctive effects have been noticed on 25 occasions.

\section{Typical Patterns of Frequency Deviations due to SC's}

A study of the frequency deviation patterns due to sudden commencements indicated that the effect may be classified into four distinct types depending on the nature of the time variation of frequency deviation, typical examples of which are shown in Fig. 1. It may be seen that in Type I (Fig. 1A) there occurs a positive frequency deviation within a few minutes from the onset of $\mathrm{SC}$, whereas in Type II (Fig. 1B) there occurs a negative frequency deviation. In type III a positive frequency shift followed by a prolonged negative frequency deviation occurs (Fig. 1C), whereas in Type IV the pattern is reversed (Fig. 1D). These four types of frequency deviation patterns due to sudden commencements have been designated as SCFD (+), SCFD (-), SCFD $(+-)$, and SCFD $(-+)$ respectively. Matsushita $(1960,1962)$ reported that the major type of sudden commencement at low latitudes is SC $(+)$ and all the $60 \mathrm{SC}$ 's used in the present study belong to this category. This leads to the observation that for one and the same type of SC, four distinct patterns of frequency deviations exist over low latitude radio paths.

\section{Statistical Results of SCFD Events}

Out of the 25 frequency deviation effects noticed in our data, 21 fit into 

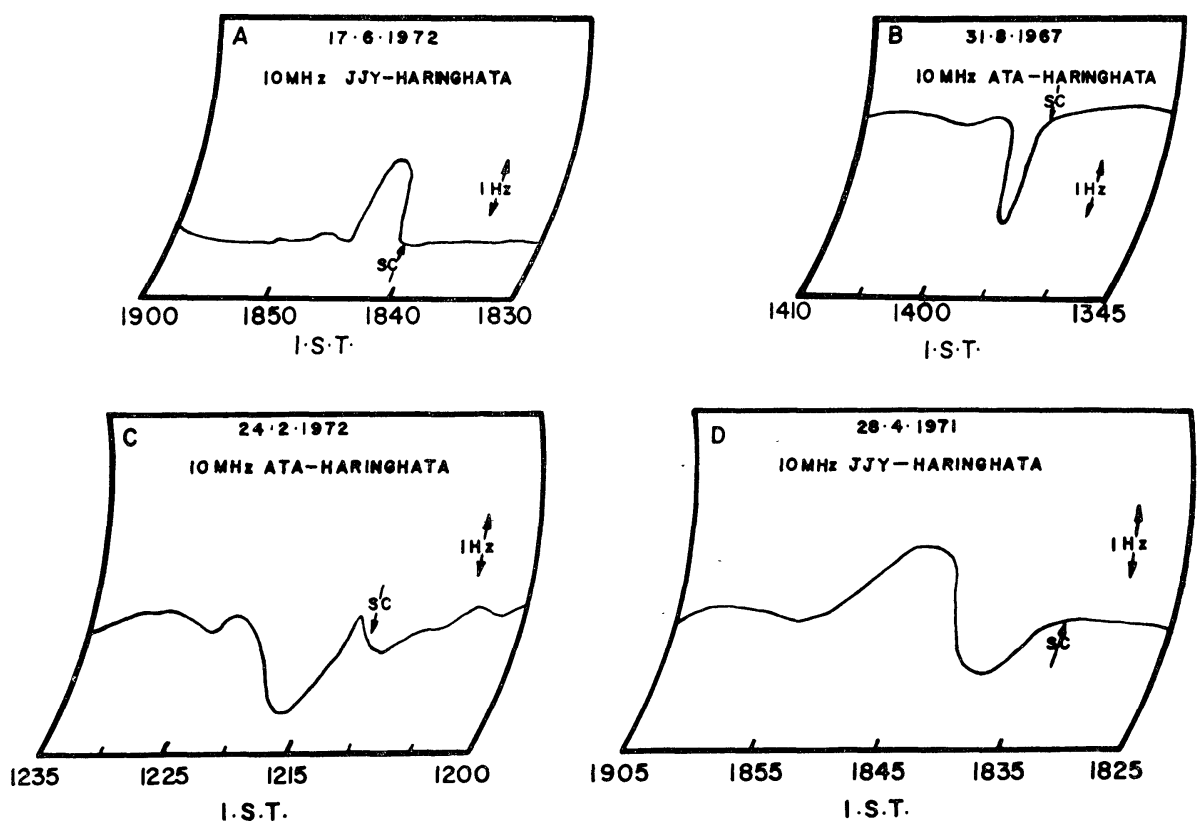

Fig. 1. Typical examples of SCFD events. A, SCFD (+); B, SCFD (-); C, SCFD $(+-) ; \mathrm{D}, \operatorname{SCFD}(-+)$.

one of the types mentioned in the preceding section while the rest four show complex variation and could not be classified. Out of the 21 classified events, 14 occurred on the JJY-Haringhata path (path length $5700 \mathrm{~km}$ ) and 7 on the ATA-Haringhata path (path length $1290 \mathrm{~km}$ ). It is noticed that out of the four types of SCFD, SCFD (-) occurs most frequently on both the paths. It is rather interesting to note from our data that SCFD $(-+)$ has never been observed on the ATA-Haringhata path (Period of monitoring 1100-1500 I.S.T. everyday.) during the entire period under study.

The total duration of the SCFD event is found to range from 2 to $20 \mathrm{~min}$ with an average value of $8.8 \mathrm{~min}$ for the sample of 21 events as can be seen from Fig. 2A. No clear cut dependence of the total duration of the SCFD event either on the amplitude of the sudden commencement or on the rise time is noticed. In composite events i.e., SCFD $(+-)$ and SCFD $(-+)$, a positive linear relationship between the maximum positive frequency deviation and maximum negative frequency deviation is noticed, (Fig. 2B). A study of the diurnal distribution of the occurrence of the different types of SCFD events showed that the most suitable period for the occurrence of Doppler frequency effects due to SC's are noon time, post-sunset and midnight periods, and that SCFD $(-+)$ is mostly a night time event. A study of the amplitude of the sudden commencement for those SC's which produced frequency deviation effects, showed that the amplitude varies over a wide range of 3 to $99 \gamma$ with 

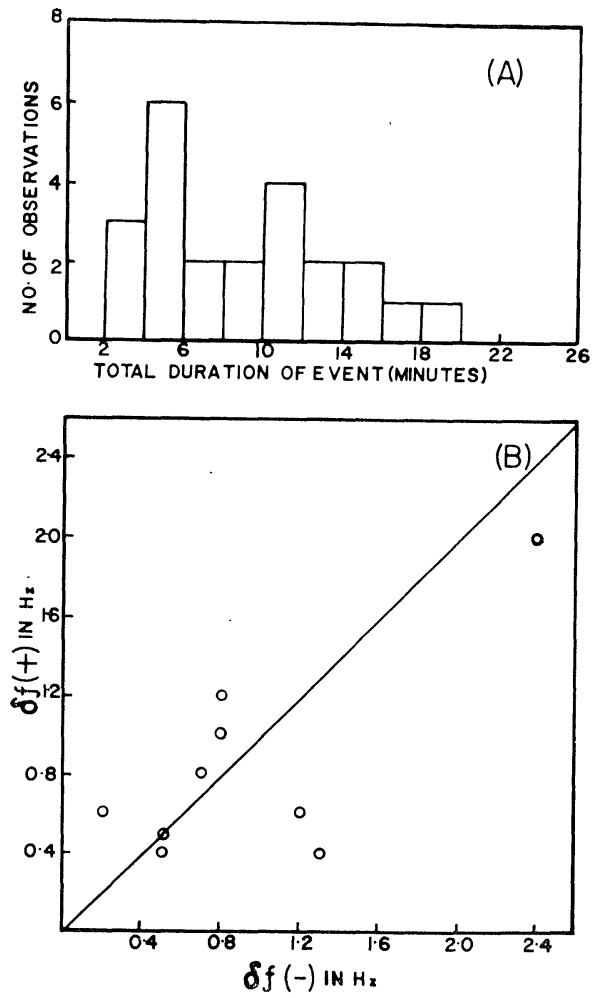

Fig. 2A. Histogram of the total duration of the SCFD event. B. Correlation between the maximum positive frequency deviation and maximum negative frequency deviation in composite events: SCFD $(+-)$ and SCFD $(-+)$.

most of the values lying in the range 10 to $40 \gamma$. No definite dependence of the type of SCFD on the amplitude of the SC is noticed. This leads to the conclusion that the magnitude of the SC has no bearing either on the occurrence or on the nature of the frequency deviation effect due to the sudden commencement.

It is observed that during the decay period, the frequency deviation closely follows the expression:

$$
\Delta f=\Delta f_{0} \exp \left(-\left(t-t_{0}\right) / \tau\right)
$$

where $\Delta f_{0}$ is the frequency deviation at some reference time to and $\tau$ is the effective relaxation time. This feature can be clearly seen from typical examples of Log $\Delta f V s$ time plots shown in Fig. 3 for SCFD (+-) and SCFD (-) events. The value of $\tau$ is found to vary over the range 100 to 400 seconds. 


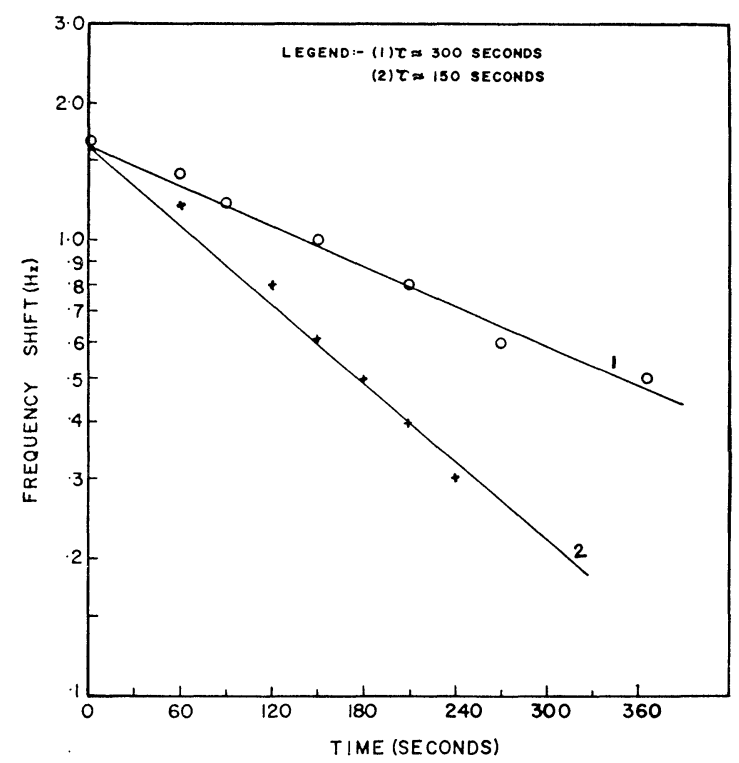

Fig. 3. Variation of negative frequency deviation with time in SCFD (+-) and SCFD (-) events.

\section{Discussion}

The earlier work on the ionospheric response to geomagnetic sudden commencements using Doppler shift technique reported by DAvies (1962) and Kanellakos and Villard (1962) showed that for the SC of September 30, 1961 , there occurred a sharp increase in frequency preceded by a definite decrease of frequency. A more detailed study using 12 SC's by CHAN et al. (1962) showed that the frequency deviation was always correlated in phase with SC $(+)$ and in opposite phase with SC $(-)$, SC $(+-)$ and SC $(-+)$. A more convincing study of the phase relationship between SC and its effect on frequency deviations has been recently made by HuANG et al. (1973) using Doppler data over a path of $150 \mathrm{~km}$ length and SC data from a place situated $95 \mathrm{~km}$ from the sub-radio reflection point. This study showed that there is no definite correlation between the phase of the SC and its effect on frequency deviations. A similar feature is noticed in the present study. However, it is to be emphasized that in this work as in that of $\mathrm{CHAN}_{\mathrm{H}}$ et al. (1962). Doppler data over long paths and geomagnetic data from a location far from the radio reflection point have been used.

A comparison of the statistical features of the SCFD events, noticed in our data with those reported by HuANG et al. (1973) showed only partial agreement. For example, the existence of four types of frequency deviation patterns for one and same type of sudden commencement, i.e., SC $(+)$, and the positive correlation between the maximum positive frequency deviation and the maxi- 
mum negative frequency deviation in composite events SCFD $(+-)$ and SCFD $(-+)$ noticed in our data agree well with the findings of HUANG et al. (1973). However, the correlation between the amplitudes of the positive spike and the amplitude of the SC reported by HUANG et al. (1973) has not been noticed in our data. It is to be emphasized that our results pertain to observations over two long radio propagation paths of different lengths $(1290 \mathrm{~km}$ and $5700 \mathrm{~km})$ while HuANG et al. (1973) used data for one short propagation path.

JACOBS and WATANABE (1966) theoretically investigated the relation between Doppler frequency changes and sudden commencements for vertically insident and reflected radio waves. A comparison of our results with those predicted by JACOBS and WATANABE (1966) using the equivalence theorem for frequency deviations could not be made due to lack of electron density profile data necessary to fix the angle of fire using ray tracing procedures. A study assuming reasonable value of ' $\theta$ ', the angle of incidence at the ionosphere, showed our results to differ from those predicted by Jacobs and Watanabe. However, much stress cannot be given to this in the absence of precise information about the propagation conditions for the events observed over a period of several years.

None of the frequency deviation patterns due to sudden commencements in our data showed wavelike oscillations as reported earlier by DAVIEs (1962), Kanllakos and Villard (1962). To sum up, the present study showed that the effect of geomagnetic sudden commencements of the SC (+) type on low latitude ionosphere manifests as sudden frequency deviations which can be classified as on the types: SCFD (+), SCFD (-), SCFD $(+-)$. The magnitude of the sudden commencement has no bearing either on the occurrence or on the nature of the frequency deviation effect. The detailed statistical features of the SCFD events seem to depend on the path length.

The authors are thankful to Dr. B.J. Srivastava, Hyderabad Geomagnetic Observatory, Hyderabad for providing the Storm sudden commencement data. The research work reported in this paper has been supported by PL-480 Project of ESSA-USA, under contract No. E-2-68 (N).

\section{REFERENCES}

AGY, V, D.M. BAKER, and R.M. Jones, Studies of solar flare effects and other ionospheric disturbances with a high frequency Doppler technique: NBS Tech. Note 306, 1965.

Chan, K.L., D.P. Kanellakos, and O.G. Villard, Jr., Correlation of short period fluctuations of the earth's magnetic field and instantaneous frequency measurements, J. Geophys. Res., 67, 2066-2072, 1962.

DAvies, K., Ionospheric effects associated with the solar flare of September 28, 1961, Nature, 193, 763-764, 1962.

Fenwick, R.C. and O.G. Villard, Jr., Continuous recordings of the frequency variation of the WWV-20 signal after propagation over a $4000 \mathrm{~km}$ path, J. Geophys. Res., 65, 3249-3260, 1960. 
Huang, Y.N., K. NAGita, and P. Yuen, The ionospheric effects of geomagnetic sudden commencements as measured with an HF sounder at Hawaii, J. Atmos. Terr. Phys., 35, 173-181, 1973.

JACobs, J.A. and T. WAtAnABE, Doppler frequency changes in Radio waves propagating through a moving ionosphere, Radio Sci., 1, 257-264, 1966.

Kanellakos, D.P. and O.G. Villard, Jr., Ionospheric disturbances associated with the solar flare of September 28, 1961, J. Geophys. Res., 67, 2265-2277, 1962.

Matsushita, S., Studies on sudden commencements of geomagnetic storms using IGY data from United States Stations, J. Geophys. Res., 65, 1423-1435, 1960.

Matsushita, S., On geomagnetic sudden commencements, sudden Impulses and Storm durations, J. Geophys. Res., 67, 3753-3777, 1962.

OGaWA, T., Frequency variations in short-wave propagation, Proc. IRE, 46, 1934-1939, 1958.

Watts, J.M. and K. DAvies, Rapid frequency analysis of fading radio signals, J. Geophys. Res., 65, 2295, 1960. 